\title{
EVALUACIÓN DE UNA INTERVENCIÓN BIOPSICOSOCIAL PARA EL MALESTAR DE LAS MUJERES EN ATENCIÓN PRIMARIA ${ }^{1}$
}

\author{
SARA VELASCO ARIAS \\ Colaboradora del Instituto de la Mujer. Ministerio de Trabajo y Asuntos Sociales \\ BEGOÑA LÓPEZ DORIGA \\ Instituto de la Mujer. Ministerio de Trabajo y Asuntos Sociales \\ MARINA TOURNÉ GARCÍA, \\ $M^{\text {a }}$ DOLORES CALDERÓ BEA, \\ INMACULADA BARCELÓ BARCELÓ y \\ CONSUELO LUNA RODRÍGUEZ \\ Servicio Murciano de Salud
}

\section{INTRODUCCIÓN}

Los trastornos mentales comunes -ansiedad, depresión y somatizaciones-, síndromes funcionales y dolor sin causa orgánica, son problemas de salud de prevalencia y relevancia creciente entre las mujeres. El informe sobre salud mental y género de la Organización Mundial de la Salud en 2002 señala que las diferencias por sexo se dan precisamente en las alteraciones metales comunes, que se presentan con doble frecuencia entre las mujeres ${ }^{2}$.

En atención primaria están alcanzando gran relevancia y causando fuerte presión asistencial. Se calcula que entre el $30 \%$ y el $60 \%$ de todas las visitas en primaria son debidas a síntomas para los que no se encuentra causa médica ${ }^{3}$,entre el $73 \%$ y el $80 \%$ de los pacientes de este tipo son mujeres-, y que resultan

1. Programa promovido y financiado por el Instituto de la Mujer, Ministerio de Trabajo y Asuntos Sociales y realizado en colaboración con el Instituto de la Mujer de la Región de Murcia y Servicio Murciano de Salud.

2. ORGANIZACIÓN MUNDIAL DE LA SAlud (OMS): Gender and women's mental health, 2002 [On line] http://www.who.int/mental_health/prevention/genderwomen/en/. También UsALL I RODIÈ, Judith.: "Diferencias de género en los trastornos del estado de ánimo: una revisión de la literatura", Abstact Española de Psiquiatría, 04 (2001), pp. 269-274.

3. PATEl, V.; ARAYA, R.; De LimA, M. et al.: "Women, poverty and common mental disorders in four restructuring societies", Social Science of Medicine, 49 (1999), pp. 1461-1471. VÁzQUEZ BARQUERO, José Luis; Wilkinson, G.; Williams, P.; Díez Manrique, J.F.; PeÑA, C.: «Mental Health and medial consultation in primary care settings», Psychological Medicine, 20 (1990), pp. 681-694. AIRZAGUE- 
relacionados, bien con diagnósticos de trastornos mentales comunes, como depresión (25\% de pacientes) ${ }^{4}$, ansiedad (38\% de los y las pacientes) ${ }^{5}$ o con somatizaciones $(58 \%)^{6}$ o bien quedan clasificados como signos y síntomas mal definidos y sin causa médica demostrable (el 13\% de los diagnósticos en mujeres en atención primaria) $)^{7}$.

La mayor importancia de estas entidades en las mujeres está relacionada con el contexto sociocultural y las vivencias provocadas por factores psicosociales de género ${ }^{8}$, por los consensos y los modelos estereotipados de roles para hombres y mujeres y los ideales de feminidad y masculinidad que imponen. De hecho, el papel de los factores de género llevó en 1988 a formular que determinadas formas de dolor y las alteraciones mentales comunes son expresiones del malestar de las mujeres ${ }^{9}$, llamándose "la otra salud mental $»^{10}$ a la que trata los sufrimientos determinados por las formas de la vida cotidiana de las mujeres. Se define así el malestar como el sufrimiento que no puede ser descifrado y expresado en palabras ${ }^{11}$ y que aparece como sufrimiento anímico o en el cuerpo como síntomas somáticos y anímicos sin causa orgánica demostrable. Agrupamos bajo el término síndromes del malestar de las mujeres las alteraciones mentales comunes, -ansiedad, depresión y síntomas somáticos-y dolores sin causa orgánica, con un enfoque que los unifica a través de su significado y propondremos intervenir en su atención bajo esta perspectiva ${ }^{11}$.

Para abordar la especificidad de este problema y con el fin de desarrollar buenas prácticas de salud con enfoque de género ${ }^{12}$ que proporcionen contenidos y métodos que sean referentes para la atención en los servicios de salud de las comunidades autónomas, el Instituto de la Mujer realizó un Programa de

NA, J.M.; Grandes, G.; Alonso-Airbiol, I.; Del Campo Chavala, J.L.; LeAGa; Fernández, M.B.; MARCO DE JUANA, J.: "Abordaje biopsicosocial de los pacientes somatizadores en las consultas de atención primaria: un estudio piloto", Atención Primaria, 29(9) (2002), pp. 558-561.

4. Organización Mundial de la SAlud: Informe de Salud en el Mundo 2001. Salud Mental: nuevos conocimientos, nuevas esperanzas. [On line] www.who.org

5. Galaverni, E.; Pozo Navarro, P.; Bellini, M.: «Estudio sobre síntomas de pánico en atención primaria", Atención Primaria, 36(6) (2005), pp. 312-316.

6. Fink, Per; Sørensen, Lisbeth; EngBerG, Marianne; Holm, Martin; Munk-JørGensen, Paul: «Utilization, and General Practitioner. Recognition Somatization in Primary Care», Psychosomatics August, 40 (1999), pp. 330-338.

7. GERVÁS, J.: "Morbilidad atendida en los servicios de atención primaria», en Onís, M. y Villar, J.: La mujer y la salud en España. Informe básico, Volumen 1, Madrid, Instituto de la Mujer, Ministerio de Trabajo y Asuntos Sociales, 1992.

8. OMS: Op. cit.

9. BURIN, Mabel: El malestar de las mujeres. La tranquilidad recetada, Buenos Aires, Paidós, 1990. TUBERT, Silvia: La sexualidad femenina y su construcción imaginaria, Madrid, El Arquero, 1988.

10. Rodríguez, Regina (ed.): El malestar silenciado. La otra salud mental, Madrid, Isis Internacional. Ediciones de las Mujeres 14, 1990.

11. VeLASCO, Sara: Atención biopsicosocial al malestar de las mujeres. Intervención en atención primaria, Madrid, Instituto de la Mujer. Ministerio de Trabajo y Asuntos Sociales, 2006. http://www. mtas.es/mujer/publicaciones/docs/Atencion\%20biopsic\%20malestar.pdf

12. VelASCO, Sara: Relaciones de género y subjetividad. Método para programas de prevención, Madrid, Instituto de la Mujer. Ministerio de Trabajo y Asuntos Sociales, 2002. http://www.mtas.es/ mujer/publicaciones/docs/Relaciones $\% 20$ de \%20genero.pdf. 
Atención Biopsicosocial al Malestar de las Mujeres desde 2003. Se diseñó un modelo de intervención y un método de atención ${ }^{13}$ que integra la naturaleza biopsicosocial y de género de estos padecimientos. Se apoya en la teoría biopsicosocial (BPS) de la salud ${ }^{13}$ con incorporación de teoría de la subjetividad ${ }^{14}$ y género ${ }^{15}$. El método se apoya en el instrumento de una relación sanitario/apaciente significativa, y desarrolla los siguientes pasos: escucha integral, localización y desciframiento del factor psicosocial desencadenante o asociado a los síntomas, señalamiento y devolución a la persona de esa localización y de la relación con la sintomatología, produciendo efectos de sujeto que lleven a que el o la paciente produzca cambios en sus circunstancias sociales y cambios en su posición subjetiva frente a ellos ${ }^{16}$. Los efectos terapéuticos sobre el cuadro clínico se esperan como producto de estos efectos de cambios psicosocial. Se excluyen de la aplicación del método pacientes con trastornos mentales graves, enfermedades de órganos vitales, consumo abusivo de tóxicos, anorexiabulimia, psicosis o tratamiento con neurolépticos y antecedentes de intentos de suicidio.

El Programa se realizó con la colaboración de las comunidades autónomas. Y, en concreto, con el Instituto de la Mujer de la Región de Murcia, el Servicio Murciano de Salud y la Gerencia de Atención Primaria de Murcia, se llevó a cabo una intervención formativo-asistencial piloto aplicando el modelo descrito. La intervención se compuso de formación de profesionales, aplicación del método BPS en las consultas individuales, sesiones clínicas BPS en centros de salud, supervisión de casos por psicólogos, grupos de apoyo de mujeres, seminarios teóricos de formación continuada en Centros de Salud (CS) y trans-

13. ENGEL, G.: "The need for a new medical model: a challenge for biomedicine», Science, 196 (1977), pp. 129-136. Tizón GARCíA, Jorge L: «A propósito del modelo biopsicosocial, 28 años después: epistemología, política, emociones y contratransferencia», Atención Primaria, 39(2) (2007), pp. 93-97. BORRELL CARRIó, Francesc; SUCHMAN, Anthony L.; EPSTEIN, Ronald M.: «The Biopsychosocial Model 25 Years Later: Principles, Practice, and Scientific Inquiry", Annals of Family Medicine (2004), pp. 576-82.

14. MeLer, Irene y TAJER, Débora (compl.): Psicoanálisis y Género. Debates desde el Foro, Buenos Aires, Lugar Editorial, 2000. BuRIN, Mabel: Género y Familia. Poder, Amor y Sexualidad en la Construcción de la Subjetividad, Buenos Aires, Paidós, 1998. GonZÁlez De CHÁveZ, Maa Asunción: Cuerpo y subjetividad femenina. Salud y género, Madrid, Siglo XXI, 1993. GonZÁlez De CHÁVEZ, Maa Asunción: Feminidad y masculinidad. Subjetividad y orden simbólico, Madrid, Biblioteca Nueva, 1998. Velasco, Sara; Ruiz, Ma Teresa; Álvarez-DARDET, Carlos: «Modelos de atención a síntomas somáticos sin causa orgánica. De trastornos fisiopatológicos al malestar de las mujeres», Revista Española de Salud Pública, 4 (80) (2006), pp. 317-333. www.msc.es/estadEstudios/publicaciones/ recursos_propios/resp/revista_cdrom/vol80/vol80_4/RS804C_317.pdf. BURIN, Mabel: Género y familia..., op. cit.

15. GijSBERG VAN WijK, C.M.T.; Vliet, Van K.P.; KolK, A.M.: "Gender perspectives and quality of care: towards appropriate and adequate health care for women", Social Science of Medicine, 43(5) (1996), pp. 707-720. MALTERUD, K.: «Symptoms as a source of medical knowledge: understanding medically unexplained disorders in women», Family Medicine, 32(9) (2000), pp. 603-611. BRINK-MUinen, A. Van Den: «Women's health care: for whom and why?», Social Science of Medicine, 44(10) (1997), pp. 1541-1551.

16. Burin, Mabel: Género y familia..., op. cit. 
misión a MIR de Medicina Familiar y Comunitaria (MFyC). De 2003 a 2006 en la Gerencia de Atención Primaria (AP) de Murcia, se impartió formación de Nivel 1 -curso de 35 horas-, dirigida a profesionales de medicina, enfermería, fisioterapia y trabajo social. Y formación de Nivel 2 -curso de 35 horas, sesiones clínicas BPS didácticas, supervisión de casos, curso de dinámica y coordinación de grupos-, con el doble objetivo de ampliar la formación práctica clínica y crear un grupo clave de profesionales que fueran capaces de coordinar sesiones clínicas, impartir seminarios en los CS y transmisión teórico práctica a MIR de Medicina Familiar y Comunitaria. Se alcanzaron 17 profesionales con Nivel 2 y 32 con Nivel 1 que participaron en la intervención piloto.

Los objetivos asistenciales del método BPS con subjetividad y género, que es objeto de la intervención, han sido: 1. Identificar los factores psicosociales asociados a los cuadros clínicos y favorecer cambios psicosociales en los y las pacientes. 2. Obtener mejoría clínica de los síntomas somáticos y anímicos y disminución del uso de psicofármacos. 3. Mejorar los indicadores indirectos de malestar psicosocial de uso de servicios e insatisfacción: disminución de la hiperfrecuentación, multiuso de servicios, multiuso de pruebas complementarias, poli-consumo de fármacos e insatisfacción percibida de pacientes y sanitarios/ as.

El presente trabajo es una evaluación de proceso, resultados e impactos del conjunto de la intervención piloto, cuyos objetivos han sido evaluar la aplicabilidad del modelo en las condiciones actuales de organización de los servicios de atención primaria, la sostenibilidad de las actividades del modelo en el tiempo, utilidad para la práctica médica y la efectividad en términos de resultados de salud de la aplicación del método clínico propuesto, medidos mediante indicadores del alcance de los objetivos asistenciales planteados para la intervención.

\section{SUJETOS Y MÉTODO}

Para el diseño y desarrollo del Programa se han tenido en cuenta criterios de aplicación de enfoque de género en programas e intervenciones en salud ${ }^{17}$. Además se han utilizado criterios sobre buenas prácticas de género acordados por la ONU en $1999^{18}$.

Se ha realizado una evaluación de proceso, resultados e impacto ${ }^{19}$. Para la evaluación del proceso de la intervención se han utilizado indicadores cuantita-

17. VelASCO, Sara: «Evolución de los enfoques de género en salud. Intersección de teorías de la salud y teoría feminista». Material docente del Diploma de Salud Pública y Género. Escuela Nacional de Sanidad, Instituto de la Mujer y Observatorio de Salud de la Mujer, 2007. http:// www.mtas.es/Mujer/programas/salud/instituto.html.

18. Acuerdo del Comité de Género de ONU sobre criterios de buenas prácticas para las mujeres y la igualdad del género (IACWGE), febrero 1999. http://www.un.org/womenwatch/resources/ goodpractices/guideline.html

19. Donabedian, A.: La calidad de la atención médica, México, La Prensa Médica Mexicana, 1991. JiMÉNEZ, Leonor et al: "Metodología para la evaluación de la calidad en instituciones de atención primaria de salud», Revista Cubana de Salud Pública, 1 (1996) http://bvs.sld.cu/revistas/spu/ vol22_1_96/spu07196.htm 
tivos de número y porcentajes de participación y actividades, e indicadores cualitativos de beneficios, aplicabilidad, sostenibilidad y dificultades. Las fuentes utilizadas han sido reuniones de seguimiento con observación participante ${ }^{20}$, cuestionario de preguntas abiertas a los y las profesionales y registros de actividad con información cualitativa, cumplimentados por los y las profesionales: sobre sesiones clínicas, supervisión de casos, seminarios teóricos y grupos de mujeres.

Para la evaluación de resultados con respecto a los objetivos asistenciales se acordaron los criterios para los indicadores entre los y las participantes; se realizó un pilotaje de recogida retrospectiva de historias clínicas de 70 casos y, posteriormente, se analizó una muestra retrospectiva de 412 historias clínicas de casos atendidos con el método BPS, recogidas por 27 profesionales (17 médicos/as, 2 enfermeras, 4 trabajadoras sociales y 4 psicólogos/a). Se utilizó un registro de datos a partir de las historias clínicas, para la construcción de indicadores cualitativos, por una parte: factores psicosociales localizados y asociados a los cuadros clínicos; cambios experimentados de posición subjetiva de los y las pacientes. Y por otra, indicadores cuantitativos de porcentaje de casos con mejoría de los síntomas somáticos y/o anímicos de los síndromes del malestar (mejoría, total, muy apreciable, moderada, escasa o nula), así como mejoría de los indicadores indirectos de malestar de uso de servicios: porcentaje de casos con desaparición de hiperfrecuentación, de multiuso de servicios y pruebas complementarias, de poli-consumo de fármacos, consumo continuado de psicofármacos y de insatisfacción.

\section{RESULTADOS}

\subsection{Resultados de proceso}

Se han realizado las siguientes actividades: 18 sesiones clínicas biopsicosociales, en las que han participado el $100 \%$ de profesionales incluidos en la intervención. Como resultados cualitativos, declaran beneficios obtenidos en cuanto a aprendizaje teórico práctico, manejo de su propio estrés, apoyo e interacción con compañeros/as para encontrar soluciones y apoyo especializado de salud mental. Se han impartido 3 seminarios en CS por 5 médicas y una trabajadora social. Se realiza un grupo de apoyo de mujeres en uno de los centros de salud, coordinado por una trabajadora social.

\subsection{Beneficios de la aplicación del modelo de atención}

Los declarados por los y las participantes de AP han sido: 1. "Aumento de percepción de lo psicosocial y de la comprensión, seguridad y satisfacción en el abordaje de los y las pacientes con malestar.»2. Aspectos nuevos añadidos para la práctica: "La relación profesional-paciente y los componentes psicosociales de género y efectos del método sobre la subjetividad." 3. "Descubrimiento del género como determinante de

20. Delgado, J.M.; GutiéRrez, J.: Métodos y técnicas cualitativas de investigación en ciencias sociales, Madrid, Síntesis, 1999. 
Sara Velasco Arias, Begoña López Doriga, Marina Tourné García, Mª Dolores Calderó...

salud y que el método biopsicosocial es aplicable también para otros problemas de la práctica clinica.» 4. "Beneficio para la vida personal por la comprensión del funcionamiento psicosocial y de género.»

La valoración que declaran los psicólogos y psicóloga de Salud Mental participantes es: 1. "Modelo de intervención biopsicosocial muy valioso para AP. Resulta asequible, flexible y aplicable, muy operativo". 2. "Modelo de relación entre Atención Primaria y Especializada basado en la cooperación, que sustituye al de derivación y que puede mostrarse efectivo y eficiente sin requerir la inversión en nuevos recursos materiales y humanos». 3. "Cambio diametral de la visión que se tiene en Salud Mental sobre el trabajo que realizan los profesionales de AP, mejora de las relaciones AP-SM». 4. "Potencial descenso de la demanda por sindromes del malestar, ya que muchos pacientes no se cronifican y no llegarán a Salud Mental.»

\subsection{Sostenibilidad}

El modelo ha resultado sostenible, ya que las personas que acceden a la formación en su totalidad aplican en la consulta el método en la medida en que el tiempo se lo permite $y$, al haber alcanzado un número crítico de profesionales de Nivel 2, se ha conseguido continuidad en la aplicación del método y han autogestionando sesiones clínicas, seminarios teóricos y grupos de mujeres, que se mantienen y continúan aún después de terminado el periodo piloto de evaluación.

\subsection{Prevalencia de síndromes del malestar}

En cuanto a la presentación clínica de los síndromes del malestar, los y las profesionales participantes estiman una prevalencia de síndromes del malestar del $30 \%$ de sus pacientes ( $80 \%$ de los cuales son mujeres).

\subsection{Aplicabilidad}

El porcentaje estimado en que están pudiendo aplicar el método, en las condiciones actuales de organización y tiempo, es el $20 \%$ de los y las pacientes que identifican con malestar en los cupos más grandes, y el $80 \%$ en los cupos más pequeños.

\subsection{Resultados de salud por aplicación clínica del método}

Se registra una muestra de 412 casos -349 de mujeres y 63 de hombres-. El $95 \%$ son declarados por médicos/as y el $5 \%$ restante por enfermería y trabajo social. El rango de edad atendido es de 8 a 91 años. No han existido límites de edad para la aplicación del modelo. Y el grupo de edad mas frecuente ha sido de 35 a 54 años ( $51,4 \%$ de los casos). Se recoge un $2 \%$ de niños/as y adolescentes.

\subsection{Cuadros clínicos}

Los más frecuentes encontrados son, para las mujeres, síntomas y síndromes funcionales $(57,9 \%)$, ansiedad $(38,4 \%)$, depresión $(37,8 \%)$ y dolor músculo- 
Evaluación de una intervención biopsicosocial para el malestar de las mujeres en...

esquelético y cefaleas $(36,1 \%$ ) (Tabla 1). Se han tratado 31 casos de fibromialgia y 3 de fatiga crónica (todos en mujeres) y 7 casos de colon irritable (1 en un hombre).

Tabla 1. Cuadros clínicos de síndromes de malestar (Multidiagnósticos)

\begin{tabular}{|c|c|c|c|c|c|}
\hline Mujeres & $\mathrm{N}^{\mathrm{o}}$ & $\%$ & Hombres & $\mathbf{N}^{\mathbf{o}}$ & $\%$ \\
\hline Ansiedad & 138 & 39,5 & Ansiedad & 20 & 31,7 \\
\hline Depresión & 132 & 37,8 & Opre. precordial/dolor torác. no card. & 12 & 19 \\
\hline Dolor musculoesquelético & 89 & 25,5 & Depresión & 11 & 17,5 \\
\hline Cansancio/ astenia & 50 & 14,3 & Insomnio / dificultad para dormir & 11 & 17,5 \\
\hline Cefalea / jaqueca / migraña & 46 & 13,2 & Cefalea / jaqueca / migraña & 10 & 15,9 \\
\hline Mareos / vértigos & 43 & 12,3 & Palpitaciones / taquicardia & 10 & 15,9 \\
\hline Insomnio / dificultad para dormir & 38 & 10,9 & Cansancio/ astenia & 7 & 11,1 \\
\hline Palpitaciones / taquicardia & 37 & 10,6 & Dolor musculoesquelético & 6 & 9,5 \\
\hline Fibromialgia & 31 & 8,9 & Crisis de pánico / angustia & 6 & 9,5 \\
\hline Opre. precordial/dolor torác. no cardia. & 24 & 6,9 & Disnea / dificultad para respirar & 6 & 9,5 \\
\hline Tristeza / decaimiento & 22 & 6,3 & Mareos / vértigos & 5 & 7,9 \\
\hline Malestar psicológico & 18 & 5,2 & Tristeza / decaimiento & 5 & 7,9 \\
\hline Crisis de pánico / angustia & 17 & 4,9 & Fobias & 5 & 7,9 \\
\hline Disminución del apetito & 16 & 4,6 & Malestar psicológico & 4 & 6,3 \\
\hline Otras Somatizaciones & 16 & 4,6 & Disminución de la libido & 3 & 4,8 \\
\hline Epigastrálgia & 13 & 3,7 & Náuseas/vómitos & 3 & 4,8 \\
\hline Disnea / dificultad para respirar & 11 & 3,2 & Alteración de la concentración & 3 & 4,8 \\
\hline Síntomas digestivos & 11 & 3,2 & Epigastrálgia & 2 & 3,2 \\
\hline Dispepsia... & 11 & 3,2 & Síntomas digestivos & 2 & 3,2 \\
\hline Problemas ginecológicos & 8 & 2,3 & Trastorno ánimo/cambios de humor & 2 & 3,2 \\
\hline Fobias & 7 & 2 & Pérdida de peso & 2 & 3,2 \\
\hline Colon Irritable & 7 & 2 & Parestesia & 2 & 3,2 \\
\hline Disminución de la libido & 4 & 1,1 & Disminución del apetito & 1 & 1,6 \\
\hline Trastorno del ánimo /cambios de humor & 4 & 1,1 & Otras Somatizaciones & 1 & 1,6 \\
\hline Miedos & 4 & 1,1 & Dispepsia... & 1 & 1,6 \\
\hline Crisis hipertensiva & 4 & 1,1 & Colon Irritable & 1 & 1,6 \\
\hline Prurito & 4 & 1,1 & Miedos & 1 & 1,6 \\
\hline Dolor abdominal & 3 & 0,9 & Crisis hipertensiva & 1 & 1,6 \\
\hline Fatiga crónica & 3 & 0,9 & Dolor abdominal & 1 & 1,6 \\
\hline Pérdida de peso & 2 & 0,6 & Alteración del sueño & 1 & 1,6 \\
\hline Parestesia & 2 & 0,6 & Fibromialgia & 0 & 0 \\
\hline Manías y obsesiones & 2 & 0,6 & Problemas ginecológicos & 0 & 0 \\
\hline Agresividad & 2 & 0,6 & Prurito & 0 & 0 \\
\hline Fallos de memoria & 2 & 0,6 & Fatiga crónica & 0 & 0 \\
\hline Bulimia & 2 & 0,6 & Manías y obsesiones & 0 & 0 \\
\hline
\end{tabular}


Sara Velasco Arias, Begoña López Doriga, Marina Tourné García, Mª Dolores Calderó...

\begin{tabular}{|l|c|c|l|c|c|}
\hline Náuseas/vómitos & 1 & 0,3 & Agresividad & 0 & 0 \\
\hline Alteración del sueño & 1 & 0,3 & Fallos de memoria & 0 & 0 \\
\hline Consumo de tóxicos & 1 & 0,3 & Bulimia & 0 & 0 \\
\hline Intento suicidio & 1 & 0,3 & Consumo de tóxicos & 0 & 0 \\
\hline Pesadillas nocturnas & 1 & 0,3 & Intento suicidio & 0 & 0 \\
\hline Baja autoestima & 1 & 0,3 & Pesadillas nocturnas & 0 & 0 \\
\hline Síndrome de adaptación & 1 & 0,3 & Baja autoestima & 0 & 0 \\
\hline Crisis de identidad & 1 & 0,3 & Síndrome de adaptación & 0 & 0 \\
\hline Bolo histérico & 1 & 0,3 & Crisis de identidad & 0 & 0 \\
\hline Síndrome de rumiación & 1 & 0,3 & Bolo histérico & 0 & 0 \\
\hline Alteración de la concentración & 1 & 0 & Síndrome de rumiación & 0 & 0 \\
\hline
\end{tabular}

Fuente: registro de casos Murcia 2006.

Para los hombres, los más frecuentes son también los síntomas somáticos $(58,7 \%)$, aunque no se encuentran ente ellos síndromes funcionales, sino principalmente somatizaciones de tipo cardiovascular (opresión precordial, dolor torácico no cardiaco y palpitaciones) y ansiedad (41,3\%), incluso más que en las mujeres y los síntomas anímicos (41,3\%). Varía en los hombres la menor frecuencia de dolor $(22,2 \%)$ y especialmente la depresión $(17,5 \%)$ con la mitad de frecuencia que en mujeres.

De 8 a 24 años, en hombres, la proporción de ansiedad y síntomas somáticos (teniendo en cuenta que muchos de ellos son cortejo somático de la propia ansiedad), son bastante más frecuentes que en edades más avanzadas. En los jóvenes son hasta 3 veces más frecuentes que para ellas los síntomas de tipo cardiovascular (25\% en chicos, $7,7 \%$ en chicas) y los digestivos $37,5 \%$ en chicos, $23,1 \%$ en chicas) y el doble de otras somatizaciones ( $25 \%$ en chicos, $11,5 \%$ en chicas). Ellas, sin embargo, presentan con más frecuencia otros síntomas anímicos variados (57,7\% en chicas y $25 \%$ en chicos). En los siguientes grupos de edad, aún manteniéndose las somatizaciones y la ansiedad en cabeza en ambos sexos, va aumentando en mujeres la importancia relativa de la depresión y los dolores músculo-esqueléticos y aparecen la fibromialgia y fatiga crónica, que son mucho más frecuentes en mujeres que en hombres a todas las edades (Figura 1).

La comorbilidad encontrada entre los diversos cuadros es del $40 \%$ en mujeres y $25 \%$ en hombres. En los casos de fibromialgia, fatiga crónica y colon irritable (comorbilidad en el 92,5\%) ha sido mayor que en los demás cuadros.

\subsection{Factores psicosociales}

Son aquellos que han tenido significado subjetivo enfermante para cada paciente (Tabla 2) y se han clasificado para su análisis en categorías que agrupen acontecimientos con similar significado psicosocial y sensibles al análisis de género. 
Evaluación de una intervención biopsicosocial para el malestar de las mujeres en...

Figura 1. Diagnósticos en mujeres y hombres por grupos de edad. Base muestral 349 mujeres y 63 hombres. Multidiagnósticos

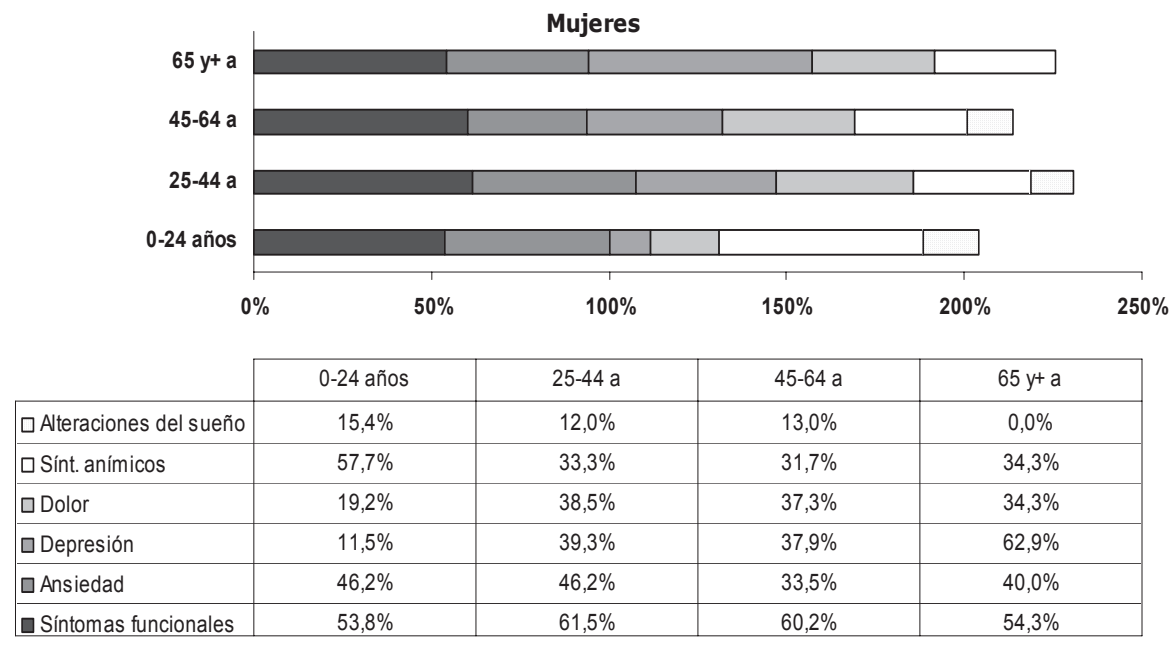

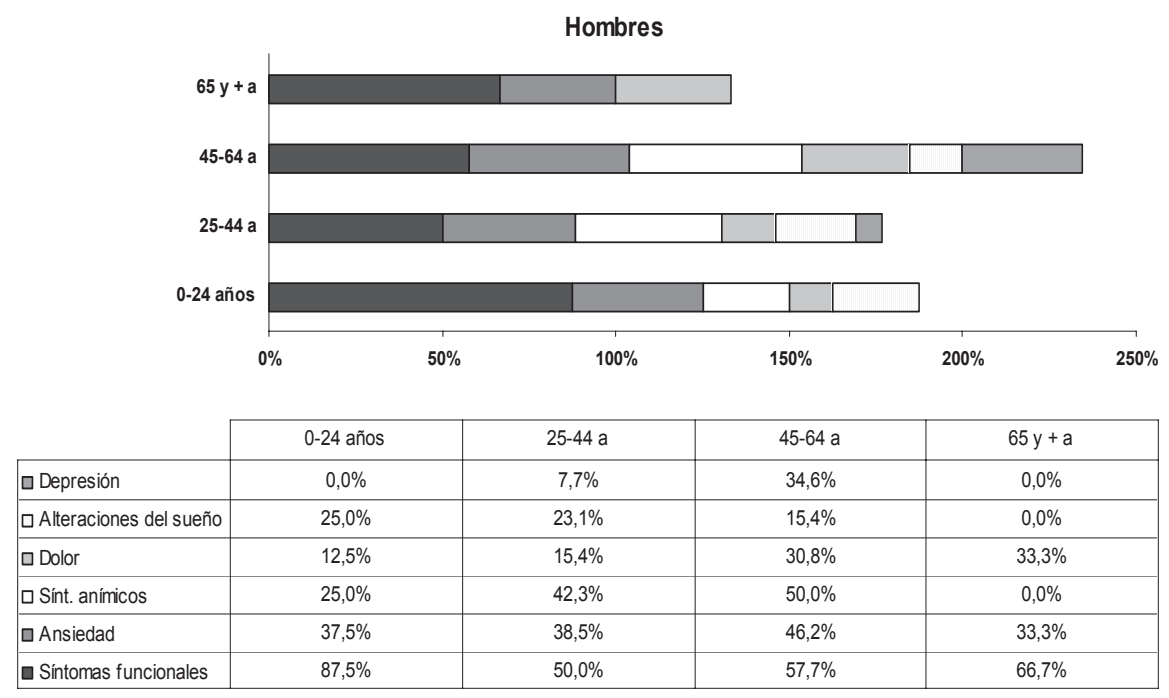

Los principales determinantes psicosociales para las mujeres son las cargas del rol tradicional -incluyendo sus efectos de dependencia, aislamiento y falta de red social- y el trabajo de cuidadora $(27 \%)$, seguido de los conflictos de pareja (20\%). Los conflictos con, o enfermedades de hijos/as $(15,3 \%)$ son el tercer factor para las mujeres. Siguen los duelos en cuarto lugar (12,3\%). Los malos tratos por la pareja o expareja, son el $6,5 \%$ de los casos de mujeres (se han descrito 26 casos de malos tratos en mujeres y 1 en un hombre) y tiene 
Sara Velasco Arias, Begoña López Doriga, Marina Tourné García, Mª Dolores Calderó...

Tabla 2. Acontecimientos psicosociales localizados. Base muestral 63 hombres y 349 mujeres

\begin{tabular}{|c|c|c|c|c|c|c|}
\hline & \multicolumn{2}{|c|}{ Hombres } & \multicolumn{2}{|c|}{ Mujeres } & \multicolumn{2}{|c|}{ Total } \\
\hline & $\mathbf{n}^{\circ}$ & $\%$ & $\mathrm{n}^{\circ}$ & $\%$ & $\mathbf{n}^{\circ}$ & $\%$ \\
\hline CONFLICTOS DE PAREJA Y ROL TRADICIONAL & 22 & $34,9 \%$ & 130 & $37,2 \%$ & 152 & $36,9 \%$ \\
\hline Conflictos de relación de pareja & 8 & $12,7 \%$ & 52 & $14,9 \%$ & 60 & $14,6 \%$ \\
\hline Separación de la pareja & 6 & $9,5 \%$ & 24 & $6,9 \%$ & 30 & $7,3 \%$ \\
\hline Hábitos tóxicos (ellos). De la pareja (ellas) & 5 & $7,9 \%$ & 21 & $6,0 \%$ & 26 & $6,3 \%$ \\
\hline Dependencia y aislamiento & 1 & $1,6 \%$ & 18 & $5,2 \%$ & 19 & $4,6 \%$ \\
\hline Sobrecarga por rol tradicional & 1 & $1,6 \%$ & 8 & $2,3 \%$ & 9 & $2,2 \%$ \\
\hline Boda próxima & 1 & $1,6 \%$ & 4 & $1,1 \%$ & 5 & $1,2 \%$ \\
\hline Carencia de red social & 0 & $0,0 \%$ & 3 & $0,9 \%$ & 3 & $0,7 \%$ \\
\hline MALOS TRATOS DE LA PAREJA & 1 & $1,6 \%$ & 26 & $7,4 \%$ & 27 & $6,6 \%$ \\
\hline $\begin{array}{l}\text { PERSONAS A SU CARGO/ENFERM. DE } \\
\text { FAMILIARES. CUIDADO }\end{array}$ & 15 & $23,8 \%$ & 141 & $40,4 \%$ & 156 & $37,9 \%$ \\
\hline Problemas de hijos/as y relación con ellos & 1 & $1,6 \%$ & 47 & $13,5 \%$ & 48 & $11,7 \%$ \\
\hline Personas inválidas o incapacitadas a su cargo & 2 & $3,2 \%$ & 38 & $10,9 \%$ & 40 & $9,7 \%$ \\
\hline $\begin{array}{l}\text { Accidentes, enfermedades, operaciones de algún } \\
\text { familiar }\end{array}$ & 3 & $4,8 \%$ & 19 & $5,4 \%$ & 22 & $5,3 \%$ \\
\hline Sobrecarga de cuidadora & 4 & $6,3 \%$ & 16 & $4,6 \%$ & 20 & $4,9 \%$ \\
\hline Enfermedades de hijos/as & 4 & $6,3 \%$ & 14 & $4,0 \%$ & 18 & $4,4 \%$ \\
\hline Conflictos de las personas a su cargo & 1 & $1,6 \%$ & 6 & $1,7 \%$ & 7 & $1,7 \%$ \\
\hline Otras referencias a personas a su cargo & 0 & $0,0 \%$ & 1 & $0,3 \%$ & 1 & $0,2 \%$ \\
\hline DOBLE JORNADA LABORAL & $\mathbf{0}$ & $0,0 \%$ & 15 & $4,3 \%$ & 15 & $3,6 \%$ \\
\hline DUELO & 13 & $20,6 \%$ & 47 & $13,5 \%$ & 60 & $14,6 \%$ \\
\hline Muerte de una familiar o allegado & 7 & $11,1 \%$ & 19 & $5,4 \%$ & 26 & $6,3 \%$ \\
\hline Muerte de la pareja & 2 & $3,2 \%$ & 16 & $4,6 \%$ & 18 & $4,4 \%$ \\
\hline Suicidio o intento de suicidio en el núcleo familiar & 3 & $4,8 \%$ & 4 & $1,1 \%$ & 7 & $1,7 \%$ \\
\hline Muerte de un hijo/a & 1 & $1,6 \%$ & 3 & $0,9 \%$ & 4 & $1,0 \%$ \\
\hline Otras referencias a pérdidas & 0 & $0,0 \%$ & 5 & $1,4 \%$ & 5 & $1,2 \%$ \\
\hline PROBLEMAS LABORALES O ESTUDIOS & 13 & $20,6 \%$ & 31 & $8,9 \%$ & 44 & $10,7 \%$ \\
\hline Problemas laborales & 8 & $12,7 \%$ & 18 & $5,2 \%$ & 26 & $6,3 \%$ \\
\hline Exámenes & 1 & $1,6 \%$ & 6 & $1,7 \%$ & 7 & $1,7 \%$ \\
\hline Acoso en el trabajo (mobbing) & 3 & $4,8 \%$ & 3 & $0,9 \%$ & 6 & $1,5 \%$ \\
\hline Problemas escolares & 0 & $0,0 \%$ & 1 & $0,3 \%$ & 1 & $0,2 \%$ \\
\hline Acoso escolar & 1 & $1,6 \%$ & 3 & $0,9 \%$ & 4 & $1,0 \%$ \\
\hline CONFLICTOS FAMILIARES & 3 & $4,8 \%$ & 7 & $2,0 \%$ & 10 & $2,4 \%$ \\
\hline Conflictos familiares & 2 & $3,2 \%$ & 5 & $1,4 \%$ & 7 & $1,7 \%$ \\
\hline Separación de los padres & 1 & $1,6 \%$ & 2 & $0,6 \%$ & 3 & $0,7 \%$ \\
\hline OTROS & 10 & $15,9 \%$ & 37 & $10,6 \%$ & 47 & $11,4 \%$ \\
\hline Inmigración & 1 & $1,6 \%$ & 9 & $2,6 \%$ & 10 & $2,4 \%$ \\
\hline Accidentes, enfermedades u operaciones propias & 5 & $7,9 \%$ & 5 & $1,4 \%$ & 10 & $2,4 \%$ \\
\hline Dificultades reproductivas & 1 & $1,6 \%$ & 5 & $1,4 \%$ & 6 & $1,5 \%$ \\
\hline
\end{tabular}


Evaluación de una intervención biopsicosocial para el malestar de las mujeres en...

\begin{tabular}{|l|c|c|c|c|c|c|}
\hline Abuso sexual & 0 & $0,0 \%$ & 3 & $0,9 \%$ & 3 & $0,7 \%$ \\
\hline Conflictos sexualidad & 1 & $1,6 \%$ & 2 & $0,6 \%$ & 3 & $0,7 \%$ \\
\hline Escasos recursos económicos & 0 & $0,0 \%$ & 1 & $0,3 \%$ & 1 & $0,2 \%$ \\
\hline Otros acontecimientos & 2 & $3,2 \%$ & 12 & $3,4 \%$ & 14 & $3,4 \%$ \\
\hline Sin respuesta & $\mathbf{2}$ & $\mathbf{3 , 2} \%$ & $\mathbf{5}$ & $\mathbf{1 , 4} \%$ & $\mathbf{7}$ & $\mathbf{1 , 7 \%}$ \\
\hline Base muestral & $\mathbf{6 3}$ & & $\mathbf{3 4 9}$ & & $\mathbf{4 1 2}$ & \\
\hline
\end{tabular}

Fuente: Registro de casos Murcia 2006.

para ellas prácticamente la misma frecuencia que los conflictos en el trabajo o con los estudios. La doble jornada laboral afecta al 3,4\% de los casos siendo un factor exclusivo de las mujeres.

Para los hombres los conflictos de pareja, incluyendo las separaciones, es el factor más frecuente (25\%) seguido de los duelos $(20,3 \%)$ y en tercer lugar, conflictos en el trabajo o con los estudios (14,1\%). Otros factores son accidentes o enfermedades propias, junto con cargas del rol tradicional y el trabajo de cuidador 7,8\%, igual en ambos factores.

El consumo abusivo de tóxicos ha tenido distinto significado en ambo sexos; si bien el porcentaje es similar ( $6 \%$ en mujeres y $7 \%$ en hombres), en los casos recogidos de hombres el problema era que ellos eran los sujetos consumidores de tóxicos, mientras que en los casos de mujeres el problema era que sus parejas eran los consumidores (excepto un caso) (Figura 2).

Figura 2. Factores psicosociales por sexos. Base muestral 64 factores en hombres y 400 factores en mujeres

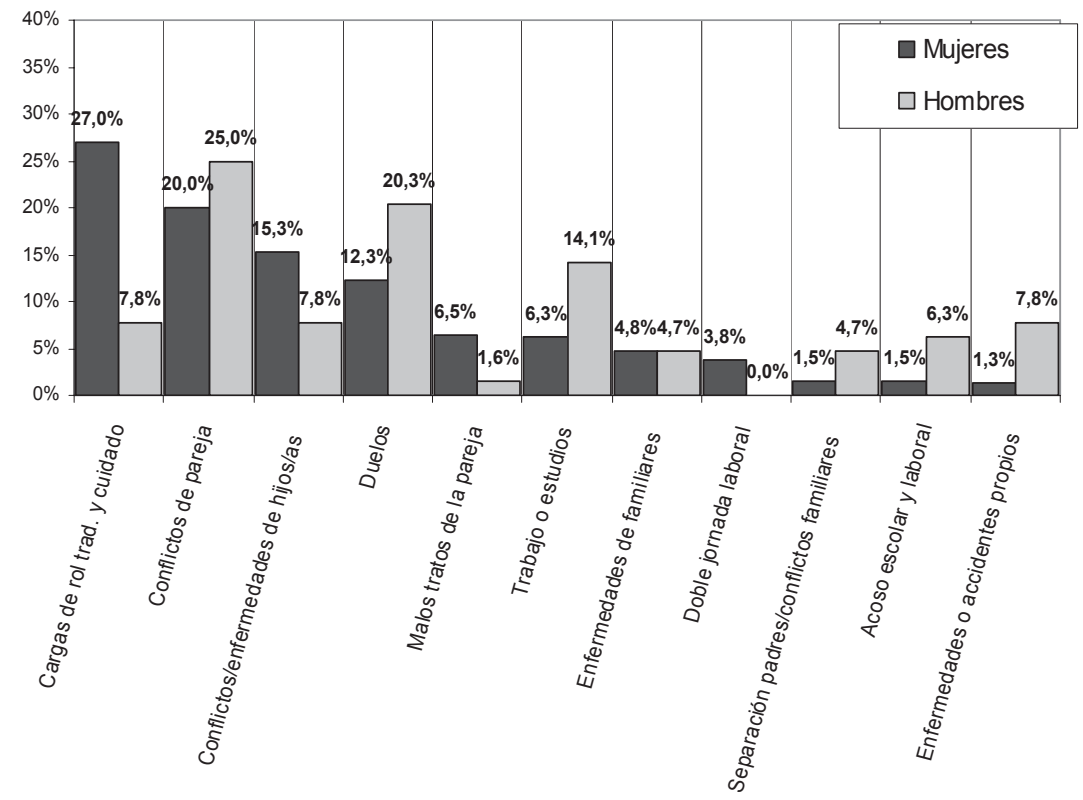

Fuente: Registro de casos Murcia 2006. 
Por grupos de edad, en niños, niñas y adolescentes, por orden de frecuencia, acoso escolar, separación de los padres y muerte de abuelos (y en un caso una amiga y la madre en otro). Para los chicos hasta 24 años, a estos factores se añaden conflictos de pareja como único otro factor. Mientras, para las jóvenes hasta 24 años, además de conflictos de pareja, aparecen la doble jornada laboral, los malos tratos y conflictos con los hijos/as.

En los siguientes grupos de edad, en las mujeres van aumentando progresivamente las cargas de rol tradicional y cuidado, los malos tratos, y los conflictos con los hijos/as y en el grupo más numeroso en la muestra, el de 45 a 64 años, en las mujeres, el $80 \%$ de los factores son sobrecarga de rol y dedicación al cuidado y soporte de los otros -cargas de rol, cuidado, conflictos de pareja, problemas con hijos/as, malos tratos y doble jornada laboral-. Mientras que para los hombres, en ese mismo grupo de edad, el $81 \%$ de los factores corresponden a conflictos de pareja, pérdidas, problemas en el trabajo o con los hijos/ as $y$ enfermedades propias.

Se ha analizado la correlación que pueda existir entre diagnósticos y factores psicosociales. Se han encontrado relaciones significativas para las mujeres. En los casos en que aparece duelo, aun sumado a otros factores, se asocia con depresión el 36,5\% de las veces, apareciendo como el más depresógeno, seguido de los malos tratos, aunque parece que en las mujeres son depresógenos cualquiera de los factores psicosociales y que cualquiera de ellos se relaciona con todas las manifestaciones del malestar. Los problemas de trabajo y estudios, sin embargo, son los que se asocian más claramente con mayores niveles de ansiedad -somatizaciones diversas $(41,2 \%)$ y ansiedad $(25,5 \%)$-, y menos con depresión $(17,6 \%)$ y dolor $(13,7 \%)$, teniendo en cuenta que es un factor más frecuente en mujeres jóvenes, también por ello más tendentes a ansiedad (Figura 3).

Figura 3. Relación entre factores psicosociales y diagnósticos en mujeres Base muestral total de factores

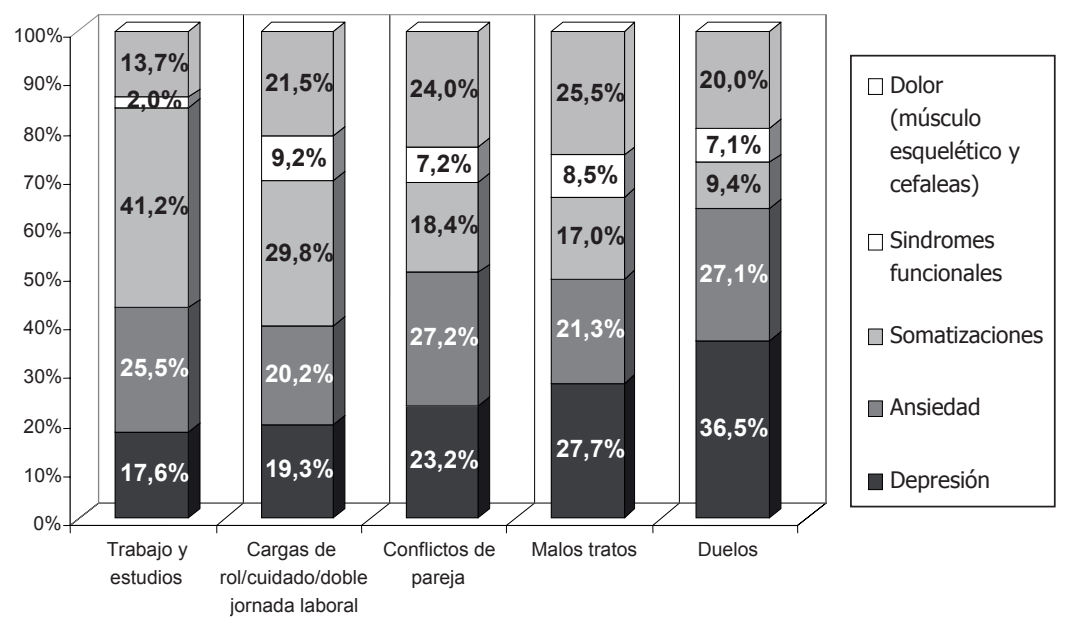

Fuente: Registro de casos de Murcia 2006. 


\subsection{Mejoría clínica}

En cuanto a la mejoría producida en los casos por la intervención BPS, se estima en base a dos conceptos: los efectos de cambio sociales y subjetivos y la mejoría clínica en sí misma. El efecto de cambio subjetivo implica que el o la paciente, mediante la localización de la circunstancia biográfica o factor psicosocial significativo y el desciframiento y señalamiento de la relación con su sintomatología, realizada con el o la profesional, se da cuenta de la posición pasiva y/o enfermante en que se encuentra e inicia cambios, tanto en sus circunstancias sociales como en la posición frente a ellas. La mejoría clínica de los síntomas y la disminución o suspensión del uso de fármacos son consecuencia de estos efectos de cambio previos. Así, se han producido efectos de cambio en el $77 \%$ de las personas atendidas, prácticamente igual en ambos sexos. Sin embargo, este resultado es bastante diferente según el nivel de formación del profesional que atiende. La eficacia de profesionales con el Nivel 2 más avanzado, es casi el doble (cambios en el $80,2 \%$ de las mujeres y el $78 \%$ de los hombres) que los de Nivel 1 (31,1\% en mujeres y un $50 \%$ en hombres).

La mejoría clínica de los síntomas experimentada por los y las pacientes es estimada por los y las profesionales declarantes basándose en el grado de desaparición de los síntomas somáticos, dolor y de los síntomas anímicos, además de la disminución o suspensión del uso de psicofármacos y analgésicos. El 71\% de los hombres y el $46 \%$ de las mujeres han mejorado totalmente (desaparecen los síntomas somáticos y anímicos y no se usan psicofármacos) o de forma muy apreciable (desaparecen los síntomas somáticos o bien los anímicos o mejoran ambos y se reduce el consumo de psicofármacos aunque no desaparezca) Se distribuyen en: $35 \%$ con mejoría total de los hombres y el $15 \%$ de las mujeres atendidas; $37 \%$ mejoría muy apreciable en hombres y $31 \%$ de las mujeres. El $3 \%$ de los hombres y $5 \%$ de las mujeres no han experimentado ninguna mejoría (Figura 4).

Figura 4. Mejoría de síntomas por sexos después de intervención BPS Base muestral 63 hombres y 349 mujeres

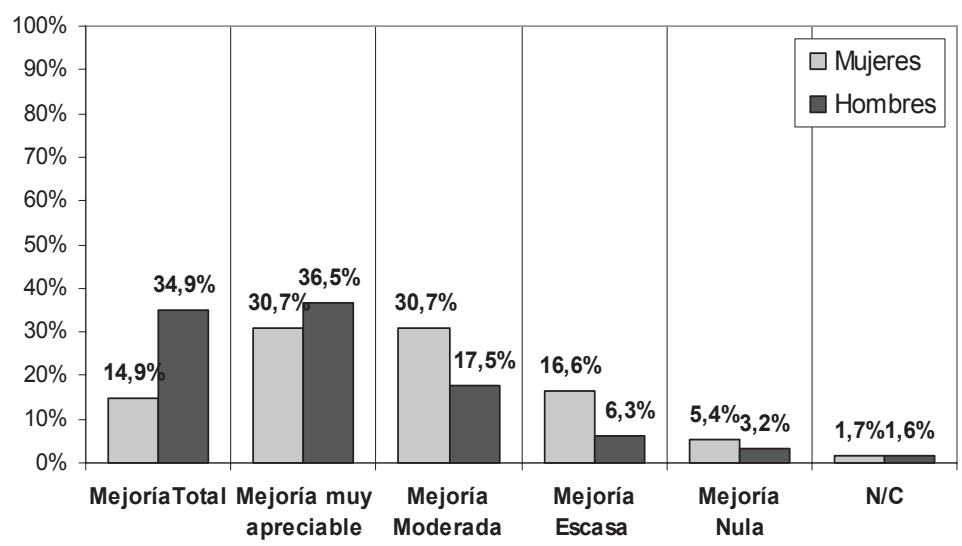

Fuente: Registro de casos de intervención en Murcia 2006. 
Sara Velasco Arias, Begoña López Doriga, Marina Tourné García, Mª Dolores Calderó...

La frecuencia de mejoría es mayor en los grupos de edad más jóvenes, tanto en hombres $(93,7 \%$ de los casos de hombres hasta 34 años han tenido mejoría total) como en mujeres (63,9\% de mejoría total en ese mismo grupo de edad). Los hombres han mejorado con más frecuencia que las mujeres en todos los grupos de edad.

De nuevo, la efectividad del método varía con el Nivel de formación. Mientras que los y las profesionales de Nivel 2, obtienen mejorías (desde totales a moderadas) en el $80,4 \%$ de los casos que tratan, los de Nivel 1 la obtienen el $66,2 \%$ de los casos.

En cuanto al consumo de psicofármacos y analgésicos y su disminución o suspensión, se produce de forma variable en todos los grados de mejoría. Del total de pacientes, el $83,5 \%$ ha disminuido o suprimido los psicofármacos y analgésicos. El $22,6 \%$ ya no precisa fármacos y el $60,9 \%$ ha disminuido su consumo.

\subsection{Distribución del grado de mejoría según entidades clínicas}

En las mujeres, la mejoría clínica se produce bastante uniformemente en relación a los diversos diagnósticos -el rango de porcentaje (de la suma de mejoría total, muy apreciable y moderada) está entre $72 \%$ y $80 \%$ para todos los diagnósticos-, a excepción de los síndromes funcionales (fibromialgia, fatiga crónica y colon irritable) que mejoran muy por debajo de la media (28\%). También se aprecia que la mejoría total es casi el doble en síntomas somáticos (14,8\%) que en depresión (8,3\%), si bien se igualan cuando se contabilizan también mejorías más moderadas.

En los hombres, se ha suprimido la categoría de síndromes funcionales (un solo caso de colon irritable que tuvo una mejoría moderada) y que produce un resultado aleatorio. En las restantes categorías se observa mejorías con frecuencia muy altas en ansiedad ( $89 \%$ de mejorías total, muy apreciable y moderada) y síntomas somáticos ( $93 \%$ de mejorías total, muy apreciable y moderada). En las demás categorías, en hombres, también son altas las frecuencias, el 91\% de las depresiones y el $79 \%$ del dolor han mejorado y sólo ha habido alguna mejoría nula en la categoría de dolor (Figura 5).

\subsection{Distribución de mejoría según factores psicosociales}

En las mujeres, la media de las mejorías en conjunto, -totales, muy apreciables o moderadas-, ha sido de $76,7 \%$. Son algo más frecuentes cuando el factor psicosocial ha sido relacionado con trabajo o estudios (93\%); en cargas de rol tradicional y carga de cuidadora $(72 \%)$ y las menores en los casos de malos tratos $(61 \%)$.

Entre los hombres, la media de mejorías en conjunto, -totales, muy apreciables o moderadas-, ha sido de $87,4 \%$. No puede considerase significativa la mejoría experimentada por malos tratos, por tratarse de un solo caso. Los demás factores tienen mejorías muy similares, con una media de mejoría total del $43,7 \%$. 
Figura 5. Grado de mejoría clínica según diagnósticos

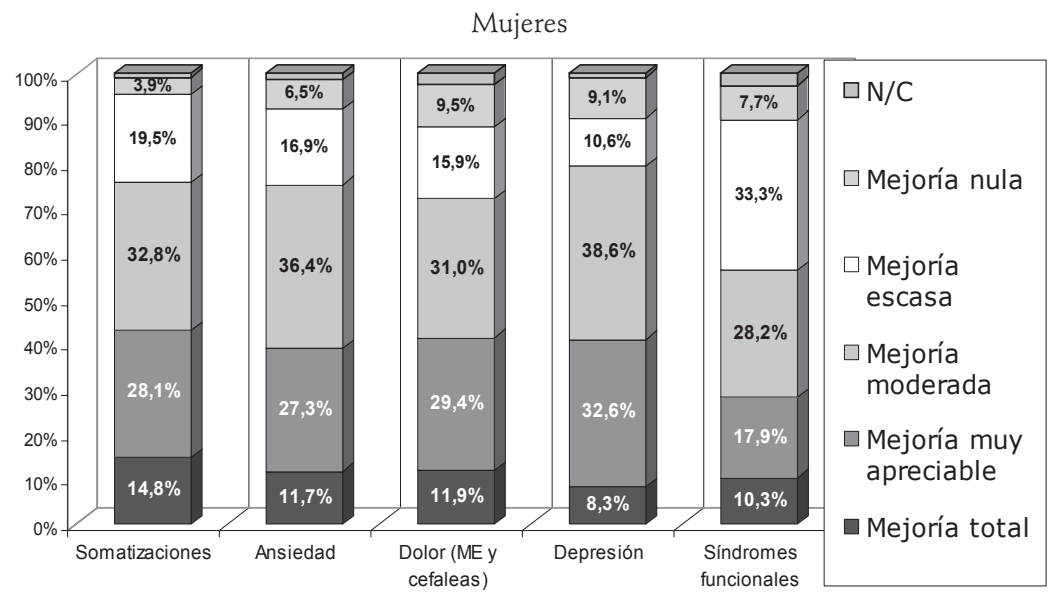

Hombres

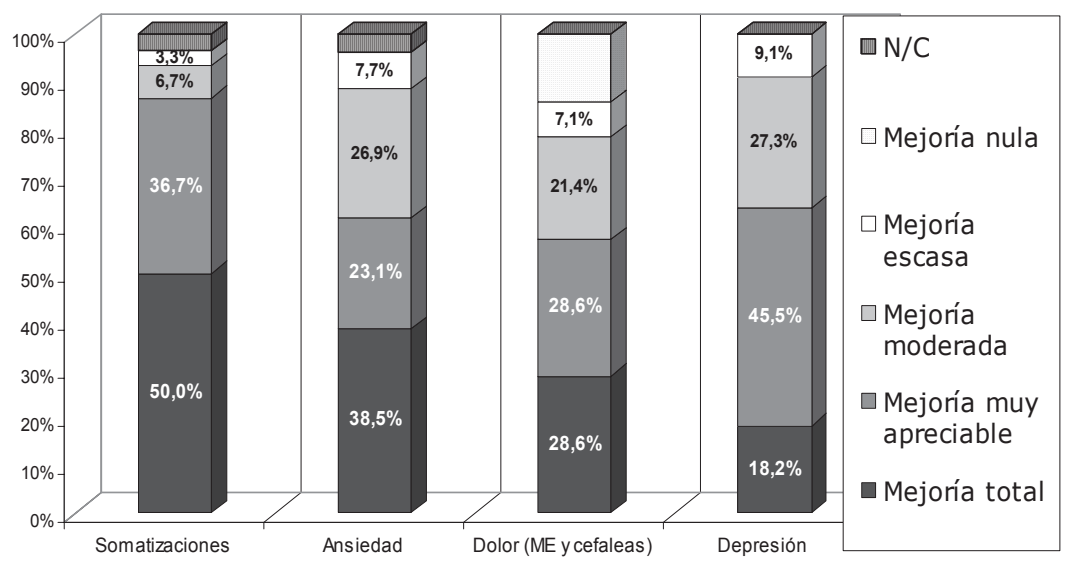

Fuente: Registro de casos de Murcia 2006.

\subsection{Indicadores de uso de recursos sanitarios}

Otro objetivo planteado era mejorar indicadores de hiperfrecuentación, multiuso de pruebas complementarias, policosumo de fármacos e insatisfacción. Algunos indicadores tienen que valorarse a medio plazo y no siempre ha constado el dato en los registros, por lo que se calculan los indicadores sobre los casos en que consta el dato antes y después de la intervención. Se produce una media de descenso del conjunto de uso de servicios y fármacos del $93 \%$ en hombres y el 77,8\% en mujeres. El 60,7\% de las mujeres y el 50,7\% de los hombres atendidos hiperfrecuentaban la consulta de medicina de familia antes de la intervención con el método BPS. Desciende la hiperfrecuentación después 
Sara Velasco Arias, Begoña López Doriga, Marina Tourné García, Mª Dolores Calderó...

Figura 6. Indicadores de uso de recursos y satisfacción de pacientes antes y después de la intervención

Hiperfrecuentación de consulta de medicina de familia antes y después de la intervención.

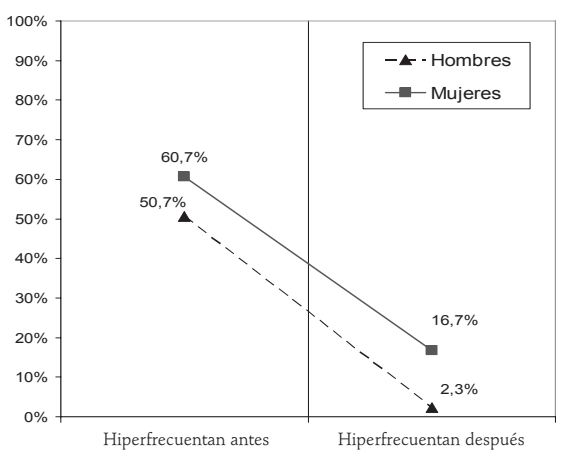

Frecuentación de otros servicios antes y después de la intervención.

Base muestrla 245 mujeres y 32 hombres

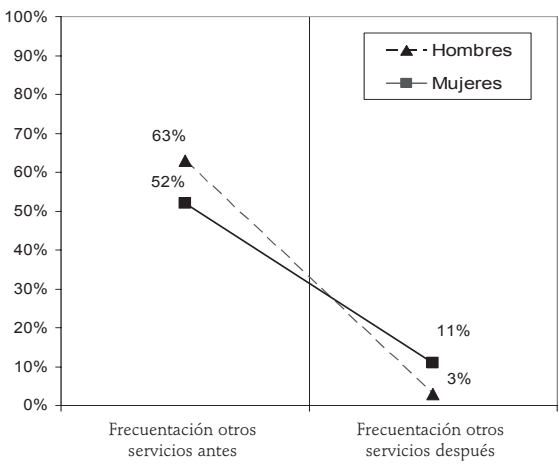

Consumo de psicofármacos antes y después de la intervención.

Base muestral 272 mujeres y 34 hombres

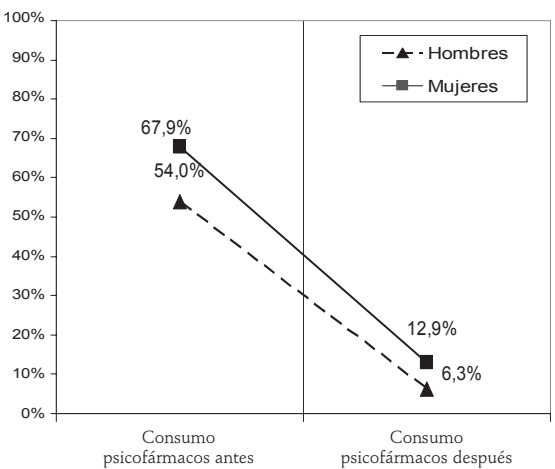

Múltiples pruebas complementarias antes y después de la intervención.

Base muestral 241 mujeres y 30 hombres

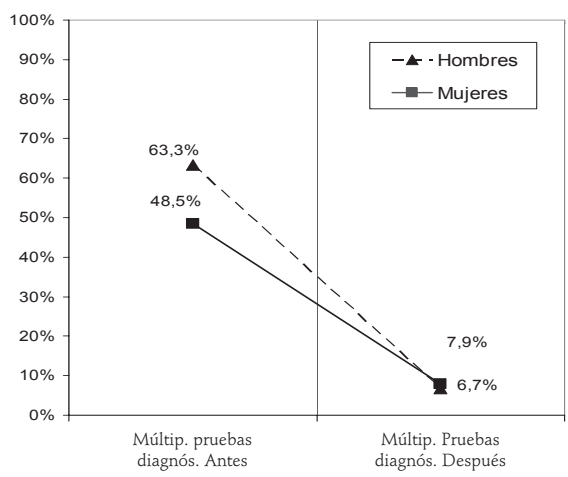

Policonsumo de fármacos antes y después de la intervención.

Base muestral 176 mujeres y 29 hombres

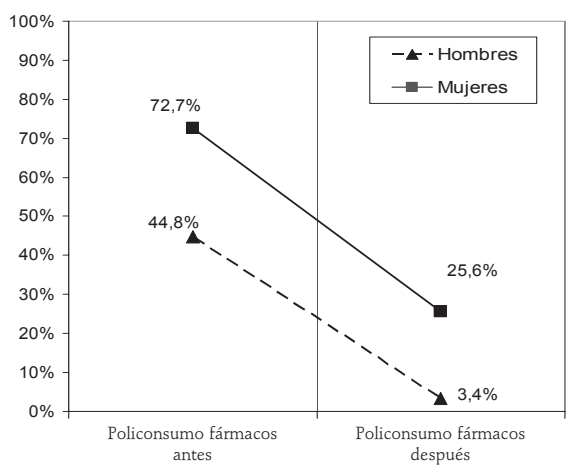

Insatisfacción y escasa eficacia de los $\mathrm{tt}^{\circ}$ antes y después de la intervención.

Base muestral 298 mujeres y 43 hombres

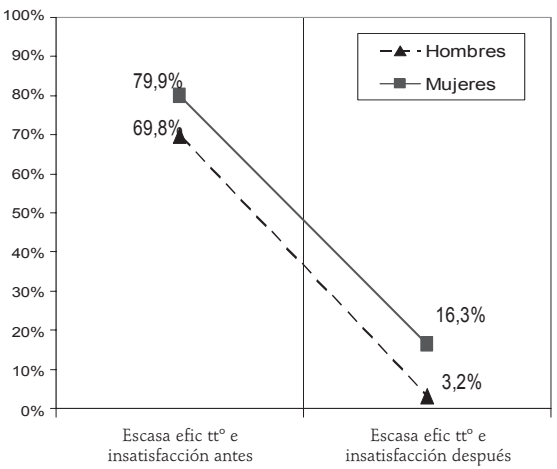


al $16,7 \%$ de las mujeres y al 2,3\% de los hombres. Frecuentaban otros servicios por encima de lo que el médico/a considera esperable para su estado de salud, el $52 \%$ de las mujeres y el $63 \%$ de los hombres. Esta frecuentación baja al 11\% en las mujeres y el $3 \%$ en los hombres. Utilizaban múltiples pruebas en más cantidad de lo que el médico/a indicaría, el 48,5\% de las mujeres y el 63,3\% de los hombres antes de la intervención y las proporciones se reducen a $7,9 \%$ en mujeres y 6,7\% en hombres después de la intervención.

Antes de la intervención, presentaban policonsumo de fármacos, -más de 10 fármacos en los últimos 6 meses-, el 72,7\% de las mujeres y el 44,8\% de los hombres y desciende al $25,6 \%$ de las mujeres y el 3,4\% de los hombres. Con consumo continuo de antidepresivos o ansiolíticos antes de la intervención, el $67,9 \%$ de las mujeres y el $54 \%$ de los hombres y desciende a $12,9 \%$ de las mujeres y $6,3 \%$ de los hombres. Y, finalmente, antes de la intervención encontraban escasamente eficaces los tratamientos e insatisfacción de la persona enferma en el 79,9\% de las mujeres y el 69,8\% de los hombres y desciende al $16,3 \%$ de las mujeres y el 3,2\% de los hombres (Figura 6).

\section{DISCUSIÓN}

La evaluación de proceso, cuyos resultados son principalmente cualitativos, muestra que, tanto el método de atención como el modelo de intervención son aplicables si se cuenta con el compromiso e impulso de las instituciones sanitarias implicadas, como ha sido el caso de esta intervención, con la formación que se ha impartido y en la medida en que los y las profesionales puedan salvar las limitaciones de disponibilidad de tiempo, que se presenta como la principal dificultad encontrada. El modelo se sostiene por sí mismo una vez iniciado, si se cuenta con profesionales comprometidos que hayan alcanzado el Nivel 2 de formación (63 horas docentes manteniendo el contacto docente, al menos, durante un año). Una limitación de esta sostenibilidad puede ser la necesidad de alcanzar este grupo inicial de profesionales sensibilizadas/os y capacitados/ as. Las personas participantes identifican valores añadidos procedentes de incorporar instrumentos de abordaje psicosocial y producción de cambios subjetivos del método; la relación profesional-paciente, aumento de la satisfacción de pacientes y profesionales y beneficios para la vida personal, además de la cooperación entre atención primaria y salud mental. Estos resultados son comunes a otras experiencia que incluyen la atención a lo psicosocial en la práctica de atención primaria ${ }^{21}$ aún de diversos cortes conceptuales psicoanalíticos o

21. Speckens Aem; Hemert, Van A.M.; Spinhoven, P.; HaWton, K.E.; BolK, J.H.; RoOijmans, H.G.M.: "Cognitive behavioural therapy for medically unexplained physical symptoms: a randomised controlled trial», British Medical Journal, 311 (1995), pp. 1328-1332. MoRRIS, R.K.; Gask, L.; Ronalds, C.; DOWnes-Grainger, E.; Thompson, H.; GoldberG, D.: "Clinical and patient satisfaction outcomes of a new treatment for somatized mental disorder taught to general practicioners", British Journal General Practice, 49 (1999), pp. 263-267. MILROD, Barbara; 
cognitivos, como el método DEPENAS ${ }^{22}$ probado, en nuestro medio, en el País Vasco.

En cuanto a la caracterización de los síndromes del malestar que se encuentra en la muestra, se han presentado casi 6 veces con más frecuencia en mujeres que en hombres en todos los grupos de edad, concordante con las estimaciones previas y justificando el abordaje específico como problema de salud de las mujeres, aunque también se incluyan los hombres afectados. Los y las profesionales han podido abordar con el método todos los tipos de trastornos mentales comunes, somatizaciones, dolor musculoesquelético y cefaleas, recogiéndolos en proporciones coherentes con las estimaciones de prevalencia existentes en primaria $^{23}$.

Las diferencias encontradas por sexos, tanto en diagnósticos como en factores psicosociales identificados, apuntan a diferencias explicables por factores de género, es decir debidas a formas de vida, roles y cargas soportadas por ambos sexos a lo largo del ciclo vital, por sujeción a modelos estereotipados de género. Las entidades más frecuentes en la muestra son las somatizaciones, seguidas de cuadros de ansiedad con diferencias por edad y sexo. Los hombres jóvenes somatizan más con síntomas tanto cardiovasculares como digestivos que, a su vez, son cortejo somático de ansiedad y que siguen siendo más frecuentes en hombres a todas las edades. Con la edad, aún manteniéndose la somatización y ansiedad en cabeza, va aumentando en mujeres, y diferenciándose de los hombres, la importancia relativa de la depresión -el doble en mujeres que en hombres ${ }^{24}-y$ los dolores músculo-esqueléticos y aparecen fibromialgia y fatiga crónica-síndromes funcionales en más del $80 \%$ en mujeres. El sentido de estas diferencias puede ser que las mujeres están presentando formas del sufrimiento más crónicas, de condición psíquica pasiva y fijadas al cuerpo, mientras que los hombres parecen tender más a manifestar formas agudas y psíquicas activas de sufrimiento como ansiedad en todas sus formas (psíquicas y somáticas).

Ha sido identificada una amplia gama de factores psicosociales asociados al malestar con discernimiento de factores con potencial explicativo de género. En el grupo de edad de 8 a 18 años, los factores identificados son acoso escolar, separación de los padres y la muerte, sobre todo, de abuelos, casi sin diferencias por sexos. Pero, al aumentar la edad hasta 24 años, si bien para los chicos sólo aparecen conflictos de pareja, en el caso de las jóvenes ya empieza el impacto

Leon, Andrew C.; Busch, Fredric; Rudden, Marie; Schwalberg, Michael; Clarkin, John et. al: "A Randomized Controlled Clinical Trial of Psychoanalytic Psychotherapy for Panic Disorder", American Journal of Psychiatry, 164(2) (2007), p. 529.

22. AiARZAGUenA, J.M.; ARIÑO, J.: «DEPENAS, hormonas y emociones: una guía práctica para el abordaje del malestar psicológico en atención primaria», Medicina de Familia, 9 (1999), pp. 310316.

23. Ministerio De SAnidad y Consumo: Estrategia de Salud Mental del Sistema Nacional de Salud, 2007. Montero, Isabel: Op. cit. GalaVerni, E.: Op. cit. FinK, Per: Op. cit. GerVÁs, J.: Op. cit.

24. OMS: Op. cit. FinK, Per: Op. cit. Montero, Isabel; Aparicio, Dolores; Gómez-Beneyto, Manuel et al.: "Género y salud mental en un mundo cambiante", Gaceta Sanitaria, 18 (Supl.1) (2004), pp. 175-181. 
de sobrecargas de género -junto a conflictos de pareja, aparece la doble jornada laboral, los malos tratos y conflictos con los hijos/as-, que seguirán aumentando en edades sucesivas. Los determinantes psicosociales son concordantes con lo descrito en estudios de género. A las mujeres les van afectando más, a lo largo del ciclo vital, las circunstancias de su vida que las colocan en un lugar subordinado, con dedicación al cuidado y soporte afectivo de los otros y en posiciones deprivadas de proyecto propio ${ }^{25}$, siendo los problemas de los otros a su cargo (incapacidad, conflictos, enfermedades, hábitos tóxicos de sus parejas...) los que se convierten en causa de su sufrimiento ${ }^{26}$, además de las sobrecargas y riesgo propios del rol subordinado tradicional, como los malos tratos y la doble jornada laboral. Tienen, sin embargo, menos peso para ellas, los factores procedentes del trabajo ${ }^{27}$ o los accidentes o enfermedades propias, es decir, lo que tendría que ver con el desarrollo de proyectos y realización propia y el cuidado de una misma ${ }^{28}$.

Los factores que enferman a los hombres se relacionan más con conflictos de pareja en la que no están subordinados y que, por tanto, pueden afrontar desde posiciones activas no estando comprometida la propia capacidad para actuar y producir cambios, lo cual puede explicar, en parte, la más rápida mejoría de los hombre sujetos a este factor; y siguen los duelos y los problemas procedentes de la esfera del trabajo, que constituye el espacio vital más investido por el ideal de masculinidad para los hombres ${ }^{29}$.

El método se ha mostrado eficiente en una media de $76,3 \%$ de las mujeres y el $90,1 \%$ de los hombres tratados (mejorías clínicas totales, muy apreciables o moderadas), para todos los diagnósticos y factores psicosociales asociados. Se ha mostrado algo más eficiente en somatizaciones y ansiedad, en ambos sexos, lo que puede tener que ver con que estos trastornos se asocian con posiciones psíquicas activas, lo que hace que se puedan producir cambios de sujeto más fácilmente. También mejoran más las personas más jóvenes, lo que puede deberse a que, precisamente en jóvenes, somatizaciones y ansiedad son las entidades más frecuentes y es menor la cronicidad de los síntomas. Así mismo, también se ha encontrado mayor frecuencia de mejoría en hombres, probablemente porque también entre ellos es más frecuente ansiedad y somatizaciones y síntomas de menor antigüedad que en las mujeres.

25. BURIN, Mabel: "Género y Psicoanálisis: Subjetividades femeninas vulnerables», en: Psicoanálisis, estudios feministas y género. http://www.psicomundo.com/foros/genero/subjetividad.htm

26. TAJER, Débora: «Cuestiones de género en la enfermedad cardiaca. Heridos corazones de mujer». http://www.pagina12.com.ar/diario/psicologia/9-64029-2006-03-09.html.

27. ARBER, Sara: «Class, Paid Employment and Family Roles: Making Sense of Structural Disadvantage, Gender and Health Status», Social Science of Medicine, 32 (4) (1991), pp. 425-436.

28. BURIN, Mabel: «Género y Psicoanálisis..., op. cit. TAJER, Débora: Op. cit.

29. BerTakis, K.D.; Helms, L.J.; Callahan, E.J.; AzARI, R.; Leigh, P.; RobBins, J.A.: «Patient gender differences in the diagnosis of depression in primary care", Journal of Women Health and Gender Based Medicine, 10 (7) (2001), pp. 689-698. BEnSING, Jozien M.; HulSMAN, Robert; SCHReURS, Karlein: "Gender differences in fatigue. Biopsychosocial factors relating to fatigue in men and women», Medical Care, 37(10) (1999), pp. 1078-1083. 
En las mujeres las mejorías son bastante parecidas para todos los cuadros -algo más eficaz en somatizaciones y ansiedad-, y los síndromes funcionales son más resistentes. Esto debe querer decir que para las mujeres deben influir en la capacidad de respuesta otras variables distintas del cuadro clínico. Los factores psicosociales que afectan a las mujeres son principalmente cargas de rol y cuidado, subordinación y pérdida de posición subjetiva en la pareja con la consecuente exposición a malos tratos y abusos, conflictos o cuidado de hijos/ as. Y estos factores de género mejoran con más dificultad porque proceden de circunstancias externas insidiosas, modificables con dificultad, porque son de impacto vivencial más profundo y más crónico. Estos factores colocan en posición de objeto de los otros, en posiciones subjetivamente pasivas e incapacitan de forma crónica para la realización de vivencias y realizaciones, sujetas a la impotencia propia de no poder influir, controlar y cambiar las situaciones que no depende de una misma. Además, suponen una sobrecarga directamente sobre la esfera afectiva -el cuidado y la implicación en los conflictos de las personas de su entorno cercano e íntimo es llamado a veces "trabajo afectivo»" que compromete en gran medida las vivencias $y$, en consecuencia, son determinantes de salud de género potentes-.

Las diferencias de mejorías obtenidas por profesionales con diferente nivel formativo permiten identificar la formación crítica necesaria para obtener buen rendimiento, que son, al menos, 63 horas docentes teórico-prácticas.

El impacto del método sobre los indicadores de hiperutilización de recursos sanitarios, consumo de fármacos e insatisfacción, indicadores indirectos de malestar psicosocial ${ }^{31}$ es muy alto y es un beneficio que se produce incluso por profesionales de Nivel 1, ya que la apertura de los contenidos de la demanda implícita y su escucha ya frena la insistencia de esa demanda ${ }^{32}$, aún antes de que se produzcan efectos clínicos.

Una limitación de la evaluación es que faltan factores referidos a clase social, trabajo y migración, aunque sí hay observaciones sobre ello, pero no sistematizadas, donde es posible que se puedan encontrar factores que expliquen diferencias de respuesta al método. Sería interesante continuar con líneas de investigación que, manteniendo el discernimiento de los determinantes de género, profundicen en la vivencia subjetiva y social de esos factores, la forma en que producen malestar y más precisiones sobre el mecanismo del método para cambiarlas.

30. FolbRE, Nancy: «The Invisible Heart: Economics and Family Values», Contemporary Sociology, 31(2) (2002), pp. 115-119.

31. INSALUD Área 9: Programa de atención a la mujer, Madrid, 1999.

32. TIZÓN, Jorge L.: «La atención primaria a la salud mental: una concreción de la atención sanitaria centrada en el consultante», Atención Primaria, 26(2) (2000), pp. 101-117. VelASCO, Sara: Atención Biopsicosocial al malestar..., op. cit. 


\section{CONCLUSIONES}

El método BPS de subjetividad y género y el modelo de intervención evaluados son aplicables y sostenibles en los CS, siempre y cuando se alcance una masa crítica de profesionales formados en el llamado Nivel 2.

Se han encontrado evidencias de que los diversos diagnósticos que se manejan como malestar de las mujeres son susceptibles de un mismo abordaje psicosocial con efectividad y de que los factores psicosociales intervienen en alguna medida en la causalidad de los síndromes del malestar, ya que al actuar sobre ellos se producen apreciables mejorías clínicas.

El método es útil para que se identifiquen en las consultas de AP los factores psicosociales potencialmente explicativos de género y proporciona instrumentos para cambiar el efecto sobre la salud de esos factores psicosociales. Así mismo, se ha mostrado efectivo en una media de $76,3 \%$ de las mujeres y el $90,1 \%$ de los hombres tratados y ha mejorado los indicadores de hiperutilización de servicios, pruebas complementarias y consumo de fármacos, además de mejorar la satisfacción de pacientes y sanitarios/as.

Son puntos críticos la escasez de tiempo disponible en las consultas de AP y el nivel crítico de formación necesaria para aplicar el método con buenos resultados.

Agradecimiento a las personas bajo cuya responsabilidad ha sido posible llevar a la práctica la intervención y evaluación en Murcia: Instituto de la Mujer de la Región de Murcia, Paloma Recio y Griselda Frapolli; Servicio Murciano de Salud, Concha Escribano, José Antonio Alarcón, Francés Molina, Maite Martínez Ros, Antonio Martínez, Susana Blanco, psicólogos/as de Salud Mental, Juan Carlos Sánchez, Sergio Canovas, Rossana López Sabater, Antonio López, y profesionales de atención Primaria que han colaborado con mucho trabajo voluntario y entusiasta, Encarnación Abril, $M^{a}$ Isabel Alcázar, Carmen Alfonso, Rosario Arnau, Josefa Belando, Ana Matilde Calvo, $\mathrm{M}^{\mathrm{a}}$ Vicenta Candela, $\mathrm{M}^{\mathrm{a}}$ Carmen Carrión, $M^{a}$ Dolores Cuevas, $M^{a}$ Carmen Elgarresta, Natividad Fernández, Virtudes Ferrer, Bernabé García, Julián García, Mª José García, José Luís Guirao, Luisa Pilar León, José Antonio Marín, Amalia Martínez, Encarna Martínez, $M^{a}$ José Martínez, Rosa $M^{a}$ Martínez, Lourdes Matas, Carmen Nieves Orcajada, Ascensión Parra, $\mathrm{M}^{a}$ Carmen Pérez, Remedios Piqueras, José Antonio Piqueras, Beatriz Ridaura, $M^{a}$ Dolores Ríos, María Ros, $M^{a}$ Fuensanta Salinas, Elena Sánchez, Mª Isabel Sánchez, Maria Sánchez, Mª Carmen Sandoval, Milagros Tomás, Pilar Valera. 